\title{
Estimation of modal correlation coefficients from background and resonant responses
}

\author{
V. Denoël† \\ National Fund for Scientific Research, University of Liège, Department of Architecture, Geology, \\ Environment and Construction, Chemin des Chevreuils, 1, Bat. B52/3, B-4000 Liège 1, Belgium
}

(Received March 27, 2008, Accepted June 4, 2009)

\begin{abstract}
A new simple relation for the estimation of modal correlation coefficients is presented. It is obtained from the decomposition of covariances of modal responses into background and resonant contributions, as it is commonly done for the variances. Thanks to appropriate assumptions, the modal correlation coefficients are estimated as weighted sums of two limit values, corresponding to the background and resonant responses respectively. The weighting coefficients are expressed as functions of the background-to-resonant ratios, which makes the proposed formulation convenient and easily accessible. The simplicity of the mathematical formulation facilitates the physical interpretation. It is for example proved that modal correlation coefficients can be non negligable even in case of well separated natural frequencies, which is sometimes unclear in the litterature. The new relation is mainly efficient in case of large finite element models. It is applied and validated on a finite element buffeting analysis of the Viaduct of Millau, the highest bridge deck ever built so far.
\end{abstract}

Keywords: modal correlation; correlation coefficient; CQC; SRSS; buffeting analysis; background response; resonant response; Viaduct of Millau.

\section{Introduction}

The dynamic analysis of slightly damped systems subjected to random low frequency excitations concerns a wide class of physical problems. Among them, a typical aspect of wind engineering only, the buffeting analysis of bridge decks, is herein investigated. Similar developments could in principle be used in other domains.

The work of Davenport $(1961,1962)$ is commonly recognized as the first application of probabilistic concepts to buffeting analysis of civil engineering structures. In these very early developments, the equations of motion were solved in a modal space and in a frequency domain. In order to avoid heavy Monte Carlo simulations inherent to time domain simulations (Rossi 2004, Carrassale 2006, Costa 2007) and to take advantage of the modal reduction, a similar approach is considered in this paper.

During the following developments (Scanlan 1978, Scanlan and Jones 1990), the single-mode approach considered by Davenport was replaced by a multi-mode approach, but without modal coupling, nor modal correlations. Because of its effectiveness and rapidity of application, this

$†$ Ph.D., Corresponding author, E-mail: v.denoel@ulg.ac.be 
method, known as the SRSS (square root of the sum of the squares) combination technique, is still widely used today. More recently Jain et al. (1996), Katsuchi et al. (1998), Chen et al. (2000), Chen et al. (2001), and Chen and Kareem (2006) have illustrated the necessity to account for modal coupling and correlation, in various contexts as buffeting analysis, aeroelastic bridge analysis and equivalent static loading. These more precise models come naturally as a strict application of the random vibration theory. They are based on the complete quadratic combination (CQC). This combination is used by Ding et al. (2002) in the context of large finite element models. They illustrate the necessity to account for modal correlations, but admit that the computational effort required for this strict application of the random vibration theory is still very heavy.

Nowadays the design of geometrically complex bridges usually relies on finite element models having a large number of degrees of freedom (DOFs). The projection of nodal forces into the modal space remains the most time consuming operation, especially when the CQC has to be applied (see Section 1.2). This is due to a strict application of the random vibration theory.

Nevertheless the effects of modal coupling and modal correlation are explained and justified exhaustively with a 2-DOF model only (e.g., Preumont 1994). From a pragmatic viewpoint, it is thus hard to believe that such heavy computations are necessary just because of the large size of the finite element model, i.e., the structural geometry, and not because of the complexity of the underlying phenomena.

This paper brings another solution to the consideration of modal correlations in buffeting analyses. It is intermediate between both extremes: (i) an unprecise but fast approach (SRSS), which neglects these correlations and is straightforward when combined with the usual decomposition of the response into background and resonant contributions and (ii) a time-consuming strict application of the random vibration theory (CQC).

In section 1, the context and nomenclature are introduced through a short recall of the random vibration theory. The innovative part of the paper is section 2 where the approached expressions for the correlation coefficients of modal responses are developed. Before some concluding comments, the proposed relation is validated with a buffeting analysis of the Viaduct of Millau.

\section{Random vibration theory}

\subsection{Strict application of the random vibration theory}

An $N$-DOF finite element model of a structure is considered. It is supposed that the power spectral density matrix (PSDM) of the nodal forces $\mathbf{S}^{(F)}(\omega)$ is known. The establishment of this input data is not the purpose of the paper. In a buffeting analysis, this matrix results from the power spectral densities of the components of wind turbulence, the wind coherence and the geometry of the structure. Details about the establishment of $\mathbf{S}^{(F)}(\omega)$ are given for example by Simiu and Scanlan (1996) or Holmes (2007) for 2-D or 3-D wind fields.

The mass $\mathbf{M}$, stiffness $\mathbf{K}$ (and therefore mode shape $\Phi$ ) and damping $\mathbf{C}$ matrices result from the finite element model (e.g., Zienkiewicz 1991). They include both structural and aeroelastic terms. Amongst available wind loading models (e.g., Denoël 2006), a quasi-steady approach is considered. Part of the damping matrix comes therefore from the aerodynamic damping. The generalized mass, stiffness and damping matrices are given by 


$$
\mathbf{M}^{*}=\Phi^{T} \mathbf{M} \Phi ; \quad \mathbf{K}^{*}=\Phi^{T} \mathbf{K} \Phi ; \quad \mathbf{C}^{*}=\Phi^{T} \mathbf{C} \Phi
$$

where the * symbolizes generalized quantities. The off-diagonal terms of these matrices are responsible for modal coupling. Because of these coupling terms, the response in a mode cannot be computed independently from the responses in the other ones. As the present developments aim at studying the effects of modal correlation only, it is supposed that the generalized matrices are diagonal. Hence, the resulting transfer function matrix

$$
\mathbf{H}^{*}(\omega)=\left(-\mathbf{M}^{*} \omega^{2}+j \omega \mathbf{C}^{*}+\mathbf{K}^{*}\right)^{-1}
$$

is also diagonal ( $j$ denotes the imaginary unit).

The analysis in the frequency domain requires the discretization of the frequency space into a set of $n_{f}$ computation frequencies, chosen in such a way to represent accurately the transfer function matrix and the PSDM of the nodal forces. Essentially a uniform distribution with supplementary computation frequencies around the natural frequencies could be sufficient (e.g., SAP2000), but more optimized distributions could also be used (Denoël 2005). For the slightly damped civil engineering structures, many computation frequencies are required to represent precisely the peaks in frequency response functions. Typically $n_{f}$ is approximately equal to 40 times the number $M$ of modes $\left(n_{f} \cong 40 M\right)$.

The first step of the stochastic analysis in the modal space is the projection of the forces into this space

$$
\mathbf{S}^{\left(F^{*}\right)}(\omega)=\Phi^{T} \mathbf{S}^{(F)}(\omega) \Phi
$$

where $T$ represents the matrix transposition. This step is the most time-consuming operation of a stochastic analysis because of the large size of $\mathbf{S}^{(F)}(\omega)(N \times N)$ and the large number $n_{f}$ of computation frequencies. As a consequence of coherence in wind forces and complexity of mode shapes, the PSDM of the generalized forces $\mathbf{S}^{\left(F^{*}\right)}(\omega)$ is usually not diagonal, which indicates a certain coherence in generalized forces.

The second step consists in the resolution of the equations of motion, i.e., the computation of the PSDM of modal responses

$$
\mathbf{S}^{(\eta)}(\omega)=\mathbf{H}^{*}(\omega) \mathbf{S}^{\left(F^{*}\right)}(\omega) \overline{\mathbf{H}^{* T}(\omega)}
$$

where the upper bar denotes the complex conjugate. Because it is supposed that the transfer function matrix $\mathbf{H}^{*}$ is diagonal, each element of $\mathbf{S}^{(\eta)}(\omega)$ is expressed by

$$
\mathbf{S}_{m n}^{(\eta)}(\omega)=\mathbf{H}_{m m}^{*}(\omega) \mathbf{S}_{m n}^{\left(F^{*}\right)}(\omega) \overline{\mathbf{H}_{n n}^{*}(\omega)}
$$

Non zero off-diagonal terms indicate that the modal responses are not uncorrelated. Their origins are the corresponding off-diagonal terms of the PSDM of generalized forces (Eq. (3)). A modal correlation signifies that the maximum values of modal responses do not occur independently in each mode. The PSDM of modal responses $\mathbf{S}^{(\eta)}(\omega)$ is the most complete response information. It contains however often too much information and simplified quantities are derived from it (e.g., Preumont 1994). For instance the covariance matrix of modal responses is obtained by its 
integration along frequencies

$$
\mathbf{v}^{(\eta)}=\int_{-\infty}^{+\infty} \mathbf{S}^{(\eta)}(\omega) d \omega_{\perp}
$$

The covariance matrices of structural displacements $\mathbf{v}^{(x)}$ and internal forces $\mathbf{v}^{(p)}$ are finally obtained by

$$
\begin{aligned}
\mathbf{v}^{(x)} & =\Phi \mathbf{v}^{(\eta)} \boldsymbol{\Phi}^{T} \\
\mathbf{v}^{(p)} & =\boldsymbol{\Phi}_{p} \mathbf{v}^{(\eta)} \boldsymbol{\Phi}_{p}^{T}
\end{aligned}
$$

where $\Phi_{p}$ represents the modal matrix associated to the considered force.

\subsection{CQC and SRSS combinations}

As they give information about the extreme values required for the structural design (e.g., Gurley 1997), the diagonal elements of $\mathbf{v}^{(x)}$ and $\mathbf{v}^{(p)}$ are major targets of a stochastic analysis. In the following, $\mathbf{v}^{(x)}$ only is considered, but the similarity of Eqs. (7) and (8) indicates that identical developments also hold for $\mathbf{v}^{(p)}$. The diagonal elements of $\mathbf{v}^{(x)}$, the variances, are expressed by

$$
v_{i i}^{(x)}=\sum_{m=1}^{M} \sum_{n=1}^{M} \Phi_{i m} \Phi_{i n} v_{m n}^{(\eta)}=\Sigma_{1}+\Sigma_{2}
$$

where the terms involving the variances and respectively the covariances of modal amplitudes are grouped

$$
\begin{gathered}
\Sigma_{1}=\sum_{m=1}^{M} \Phi_{i m}^{2} v_{m n}^{(\eta)} \\
\Sigma_{2}=2 \sum_{m=1}^{M} \sum_{n=m+1}^{M} \Phi_{i m} \Phi_{i n} \rho_{m n} \sqrt{v_{m n}^{(\eta)} v_{n n}^{(\eta)}}
\end{gathered}
$$

In this expression,

$$
\rho_{m n}=\frac{v_{m n}^{(\eta)}}{\sqrt{v_{m m}^{(\eta)} v_{n n}^{(\eta)}}}
$$

represents the correlation coefficient between the modal responses in modes $m$ and $n\left(\rho_{m n} \in[-1\right.$; $+1]$ ). Eq. (9) results from the strict application of the random vibration theory, and is known as the complete quadratic combination (CQC). Each term of the double summation $\Sigma_{2}$ involves a modal covariance, which itself results from the integral of the corresponding eut-diagonal element of the PSDM of modal amplitudes (Eq. (6)). These would not have to be computed if $\Sigma_{1}$ only was considered.

The SRSS (square root of the sum of the squares) combination technique postulates that $\Sigma_{2}$ is negligible compared to $\Sigma_{1}$. Therefore it does not require the estimation of out-diagonal elements of $\mathbf{S}^{(\eta)}$. As the computational effort is mainly related to the computation of each term of the sums, the ratio of computation times required for the the SRSS $\left(t_{S R S S}\right)$ and CQC $\left(t_{C Q C}\right)$ approaches are 
proportional to the number of terms

$$
\frac{t_{S R S S}}{t_{C Q C}} \simeq \frac{n_{f} M}{n_{f} \frac{M(M-1)}{2}}=\frac{2}{M-1^{\wedge}}
$$

\subsection{Decomposition into background and resonant components}

The SRSS combination method requires the estimation of the modal variances only (Eq. (6)). In practical applications, the definite integral required for their evaluation is avoided by writing

$$
S_{m m}^{(\eta)}(\omega)=S_{m m}^{\left(F^{*}\right)}(\omega)\left|H_{m m}(\omega)\right|^{2}=\frac{S_{m m}^{\left(F^{*}\right)}(\omega)}{K_{m m}^{* 2}}+\left(\left|H_{m m}(\omega)\right|^{2}-\frac{1}{K_{m m}^{* 2}}\right) S_{m m}^{\left(F^{*}\right)}(\omega)
$$

The second term is the product of a very sharp function in the vicinity of the circular frequency $\omega_{m}$ in mode $m$ and a slowly decreasing function. It is then commonly adopted to replace this slow varying function by a constant value, equal to the ordinate of the function at the location of the peak $\left(S_{m m}^{\left(F^{*}\right)}\left(\omega_{m}\right)\right)$. The first function (into the brackets) has to be adapted too, otherwise a non-zero asymptotic value for $\omega \rightarrow \infty$, would return an infinite variance. The diagonal elements of the PSDM of modal responses are then approached by

$$
S_{m m}^{(\eta)}(\omega)=\frac{S_{m m}^{\left(F^{*}\right)}(\omega)}{K_{m m}^{* 2}}+\left|H_{m m}(\omega)\right|^{2} S_{m m}^{\left(F^{*}\right)}\left(\omega_{m} \lambda\right.
$$

This approximation is valid for slightly damped structures and provided the energy of the loading is contained in a frequency band much lower than the natural frequencies, which is usual in case of turbulence loading. The integration along frequencies of Eq. (14) gives

$$
v_{m m}^{(\eta)}=B_{m}+R_{m}
$$

where

$$
\begin{gathered}
B_{m}=\frac{v_{m m}^{\left(F^{*}\right)}}{K_{m m}^{* 2}} \text { and } \\
R_{m}=S_{m m}^{\left(F^{*}\right)}\left(\omega_{m}\right) \int_{-\infty}^{+\infty}\left|H_{m m}(\omega)\right|^{2} d \omega=\frac{\pi \omega_{m}}{2 \xi_{m}} \frac{S_{m m}^{\left(F^{*}\right)}\left(\omega_{m}\right)}{K_{m m}^{* 2}}
\end{gathered}
$$

are the background and resonant contributions to the modal variance. The damping ratio in mode $m$ is symbolized by $\xi_{m}=C_{m m}^{*} / \sqrt{K_{m m}^{*} M_{m m}^{*}}$ and $v_{m m}^{\left(F^{*}\right)}$ denotes the variance of the generalized force in mode $m$. Thanks to these analytical relations for both components, this decomposition bypasses the heavy integration of the PSDM and hence the discretization of the frequency domain. The computation frequencies are thus limited to the list of natural frequencies $\left(\omega_{m}\right)$ plus one, since the covariance matrix of the nodal forces has to be projected into the modal space too (estimation of $v_{m m}^{\left(F^{*}\right)}$ ).

The ratio of computational efforts is thus reduced to

$$
\frac{t_{S R S S}}{t_{C Q C}} \simeq \frac{M+M}{n_{f} \frac{M(M-1)}{2}}=\frac{4}{n_{f}(M-1)}=\frac{1}{10 M(M-1)}
$$


This relation justifies the reason for which the CQC approach is considered to be time-consuming (Ding et al. 2002). The difference is now significative, even for a couple of modes.

\section{Estimation of modal covariances}

\subsection{General developments}

The covariances between modal amplitudes in different modes $v_{m n}^{(\eta)}$ present a quasi-static contribution and two higher contributions around both resonance peaks. It is thus difficult to rigorously justify the use of the same simplified procedure for the estimation of covariances. Nevertheless this paper suggests to continue using one term-only for both resonance peaks. Similarly to the developments of the previous section, this resonant term is obtained by replacing the cross-power spectral density of the generalized force by an "equivalent white noise" (whose intensity $S_{w n}$ is discussed later) and decomposing the product of transfer functions

$$
\begin{aligned}
S_{m n}^{(\eta)}(\omega) & =H_{m m}(\omega) \overline{H_{n n}(\omega)} S_{m n}^{\left(F^{*}\right)}(\omega) \\
& =\frac{S_{m n}^{\left(F^{*}\right)}(\omega)}{K_{m m}^{*} K_{n n}^{*}}+\left(H_{m m}(\omega) \overline{H_{n n}(\omega)}-\frac{1}{K_{m m}^{*} K_{n n}^{*}}\right) S_{m n}^{\left(F^{*}\right)}(\omega) \\
& \simeq \frac{S_{m n}^{\left(F^{*}\right)}(\omega)}{K_{m m}^{*} K_{n n}^{*}}+\left(H_{m m}(\omega) \overline{\left.H_{n n}(\omega)\right)} S_{w n}\right.
\end{aligned}
$$

The integration along frequencies yields the modal covariance

$$
v_{m m}^{(\eta)}=B_{m n}+R_{m n}
$$

It is also expressed as the sum of background and resonant contributions

$$
\begin{gathered}
B_{m n}=\frac{v_{m n}^{\left(F^{*}\right)}}{K_{m m}^{*} K_{n n}^{*}} \\
R_{m n}=S_{w n} \int_{-\infty}^{+\infty} H_{m m}(\omega) \overline{H_{n n}(\omega)} d \omega \\
=\frac{\pi \sqrt{\omega_{m} \omega_{n}}}{2 \sqrt{\xi_{m} \xi}} \frac{S_{w n}}{K_{m m}^{*} K_{n n}^{*}} \phi\left(\omega_{m}, \omega_{n}, \xi_{m}, \xi_{n}\right)
\end{gathered}
$$

where

$$
\phi\left(\omega_{m}, \omega_{n}, \xi_{m}, \xi_{n}\right)=\frac{8\left(\omega_{m} \omega_{n}\right)^{3 / 2}\left(\xi_{m} \omega_{m}+\xi_{n} \omega_{n}\right) \sqrt{\xi_{m} \xi_{n}}}{\left(\omega_{m}^{2}-\omega_{n}^{2}\right)^{2}+4 \omega_{m} \omega_{n}\left(\xi_{m} \omega_{m}+\xi_{n} \omega_{n}\right)\left(\xi_{m} \omega_{n}+\xi_{n} \omega_{m}\right)}
$$

is a natural frequency separation indicator because of the term $\left(\omega_{m}^{2}-\omega_{n}^{2}\right)^{2}$ appearing in the denominator. For slightly damped structures, it reaches indeed its maximum value around $\omega_{m}=\omega_{n}$, where it takes the value 


$$
\phi\left(\omega_{m}, \omega_{n}, \xi_{m}, \xi_{n}\right)=\frac{\sqrt{\xi_{m} \xi_{n}}}{\frac{\xi_{m}+\xi_{n}}{2}}
$$

which is therefore smaller than unity, and as small as $\xi_{m}$ and $\xi_{n}$ are different.

\subsection{Selection of the intensity of the equivalent white noise}

For well-separated natural frequencies $(\phi<<)$, the resonant component $R_{m n}$ is small compared to the background component $B_{m n}$. Its estimation is useless. In this case there is no correlation due to the resonant term and the estimation of the intensity of the equivalent white noise is irrelevant.

For closely spaced natural frequencies (and similar damping ratios), $\phi$ is approaching unity, and the resonant contribution must be estimated accurately, i.e., the intensity of the equivalent white noise must be chosen adequately. By considering that the limit case $\omega_{m} \rightarrow \omega_{n}$ has to be reproduced correctly and that the white noise intensity should be expressed in terms of the cross generalized force only, a mean of the ordinates at each peak has to be considered as a good approximation for $S_{w n}$. In order to simplify the subsequent developments it is decided to opt for the geometric mean

$$
S_{w n}=\sqrt{S_{m n}^{\left(F^{*}\right)}\left(\omega_{m}\right) S_{m n}^{\left(F^{*}\right)}\left(\omega_{n}\right)}
$$

By introducing the coherence function of the generalized forces

Eq. (24) is rewritten

$$
\Gamma_{m n}(\omega)=\frac{S_{m n}^{\left(F^{*}\right)}\left(\omega_{n}\right)}{\sqrt{S_{m m}^{\left(F^{*}\right)}(\omega) S_{n n}^{\left(F^{*}\right)}(\omega)}}
$$

$$
S_{w n}=\sqrt{\Gamma_{m n}\left(\omega_{m}\right) \sqrt{S_{m m}^{\left(F^{*}\right)}\left(\omega_{m}\right) S_{n n}^{\left(F^{*}\right)}\left(\omega_{m}\right)} \Gamma_{m n}\left(\omega_{n}\right) \sqrt{S_{m m}^{\left(F^{*}\right)}\left(\omega_{n}\right) S_{n n}^{\left(F^{*}\right)}\left(\omega_{n}\right)}}
$$

Since these developments are interesting when the natural frequencies are close, let us suppose (which is true in the limit case) that

$$
\begin{gathered}
S_{m m}^{\left(F^{*}\right)}\left(\omega_{m}\right) \simeq S_{m m}^{\left(F^{*}\right)}\left(\omega_{n}\right) ; \quad S_{n n}^{\left(F^{*}\right)}\left(\omega_{m}\right) \simeq S_{n n}^{\left(F^{* *}\right)}\left(\omega_{n}\right) \\
\Gamma_{m n}\left(\omega_{m}\right) \simeq \frac{\Gamma_{m n}\left(\omega_{m}\right)+\Gamma_{m n}\left(\omega_{n}\right)}{2} \overline{\bar{\Delta}} \overline{\Gamma_{m n}} \\
\Gamma_{m n}\left(\omega_{n}\right) \simeq \frac{\Gamma_{m n}\left(\omega_{m}\right)+\Gamma_{m n}\left(\omega_{n}\right)}{2} \overline{\bar{\Delta}} \overline{\Gamma_{m n}}
\end{gathered}
$$

which can also be justified by the continuity and smoothness of PSDs encountered in practical applications. None of these assumptions concerns the eventual difference in the orders of magnitude of $S_{m m}^{\left(F^{*}\right)}$ and $S_{n n}^{\left(F^{*}\right)}$, which would be erroneous otherwise. The intensity of the equivalent white noise and the expression of the resonant contribution are finally reduced to

$$
S_{w n}=\overline{\Gamma_{m n}} \sqrt{S_{m m}^{\left(F^{*}\right)}\left(\omega_{m}\right) S_{n n}^{\left(F^{*}\right)}\left(\omega_{n}\right)} \text { and }
$$




$$
R_{m n}=\frac{\pi \sqrt{\omega_{m} \omega_{n}}}{2 \sqrt{\xi_{m} \xi_{n}}} \frac{\Gamma_{m n} \sqrt{S_{m m}^{\left(F^{*}\right)}\left(\omega_{m}\right) S_{n n}^{\left(F^{*}\right)}\left(\omega_{n}\right)}}{K_{m m}^{*} K_{n n}^{*}} \phi\left(\omega_{m}, \omega_{n}, \xi_{m}, \xi_{n}\right)
$$

The combination of Eqs. (21) and (29) gives a good approximation for the covariance of modal amplitudes, no matter the proximity of natural frequencies.

\subsection{Establishment of the correlation coefficients}

The approached modal correlation coefficients are estimated through the decomposition of the variances and covariances into their respective contributions

$$
\hat{\rho}_{m n}=\frac{v_{m n}^{(\eta)}}{\sqrt{v_{m m}^{(\eta)} v_{n n}^{(\eta)}}}=\frac{B_{m n}+R_{m n}}{\sqrt{B_{m m}+R_{m m}} \sqrt{B_{n n}+R_{n n}}}
$$

In order to recast this relation into a more interpretable formulation, let us introduce:

- the background-to-resonant ratio in mode $m$

$$
b_{m}=B_{m m} / R_{m m}, \quad b_{n}=B_{n n} / R_{n n}
$$

which is a dimensionless modal indicator of the importance of the background component compared to the resonant one. A parametric study of four representative cable-stayed bridges in China (Gu et al. 2002) stated that this indicator is typically comprised between 0.2 and 0.4 for fundamental modes. It could be larger for stiffer or more damped bridges, and smaller for other bearing systems (suspension bridges for example);

- item-the background and resonant weighting coefficients (Fig. 1)

$$
\begin{gathered}
\gamma_{B}=\frac{1}{\sqrt{1+b_{m}^{-1}} \sqrt{1+b_{n}^{-1}}} \text { and } \\
\gamma_{R}=\frac{1}{\sqrt{1+b_{m}} \sqrt{1+b_{n}}}
\end{gathered}
$$
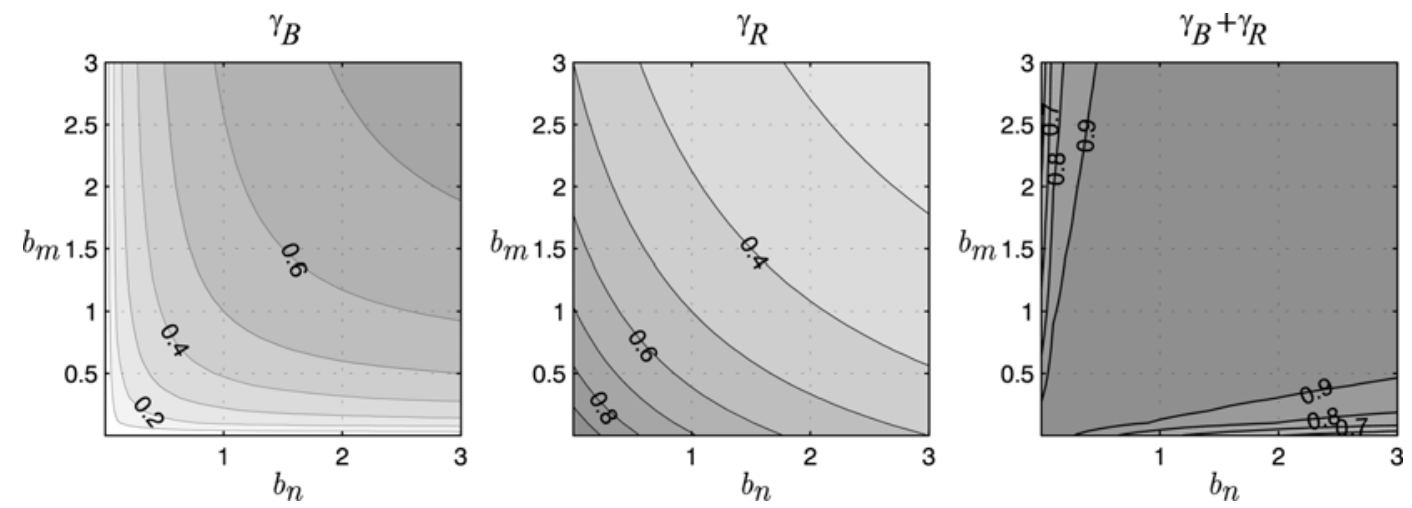

Fig. 1 Background $\left(\gamma_{B}\right)$ and resonant $\left(\gamma_{R}\right)$ weighting coefficients, as a function of the background-to-resonant ratios $\left(b_{m}, b_{n}\right)$ 
They both belong to the interval $[0 ; 1]$ and, as shown here after, quantify the dispatching of the correlation into background and resonant contributions, for both modes. For quasi-static responses in modes $m$ and $n\left(b_{m} \gg>, b_{n}>>\right), \gamma_{B}$ tends towards unity and $\gamma_{R}$ tends towards zero. On the contrary, for resonant responses in both modes $\left(b_{m}<<, b_{n}<<\right), \gamma_{B}$ tends towards zero and $\gamma_{R}$ tends towards unity.

With these notations, Eq. (30) is written

$$
\hat{\rho}_{m n}=\gamma_{B} \frac{B_{m n}}{\sqrt{B_{m m} B_{n n}}}+\gamma_{R} \frac{R_{m n}}{\sqrt{R_{m m} R_{n n}}}
$$

in which the remaining quotients are simplified by introducing Eqs. (16), (17), (21) and (29)

$$
\hat{\rho}_{m n}=\gamma_{B} \rho_{m n}^{\left(F^{*}\right)}+\gamma_{R} \overline{\Gamma_{m n}} \phi\left(\omega_{m}, \omega_{n}, \xi_{m}, \xi_{n}\right)
$$

This is the proposed formulation: the correlation coefficient of modal amplitudes is estimated by a weighted combination of the correlation coefficient of the generalized forces $\rho_{m n}^{\left(F^{*}\right)}$ and a "dynamic" correlation coefficient $\overline{\Gamma_{m n}} \phi\left(\omega_{m}, \omega_{n}, \xi_{m}, \xi_{n}\right)$. This latter one is expressed as the product of two functions (both comprised between -1 and +1 ) related to the proximity of the natural frequencies and to the coherence of the generalized forces in the frequency band around the natural frequencies of the considered modes.

As $\gamma_{B}, \gamma_{R}$ and $\gamma_{B}+\gamma_{R}$ are all smaller than unity (Fig. 1), the actual modal correlation coefficient is smaller than the maximum value of $\rho_{m n}^{\left(F^{*}\right)}$ and $\overline{\Gamma_{m n}} \phi\left(\omega_{m}, \omega_{n}, \xi_{m}, \xi_{n}\right)$. The proposed relation returns well both intuitively expected limit cases:

- background response in both modes $\left(b_{m}>>, b_{n}>>\right): \gamma_{B} \simeq 1$ and $\gamma_{R} \simeq 0$, the response is mainly quasi-static and the correlation of the modal responses is equal to that of the generalized forces.

The dynamic correlation is without any importance and the proximity of the natural frequencies is irrelevant;

- resonant response in both modes $\left(b_{m}<<, b_{n}<<\right): \gamma_{B} \simeq 0$ and $\gamma_{R} \simeq 1$, the correlation coefficient of the modal responses is equal to $\overline{\Gamma_{m n}} \phi\left(\omega_{m}, \omega_{n}, \xi_{m}, \xi_{n}\right)$, the dynamic correlation coefficient.

The accuracy of the proposed relation is subjected to conditions similar to those necessary for the decomposition of the variance: it is asymptotically exact for a damping ratio and an upper limit of the frequency content of excitation tending both towards zero. These conditions are usually met in practical applications.

Because of the estimation of $\overline{\Gamma_{m n}}$, the new relation still requires the full projection of the PSDM of nodal force into the modal space. Compared to the CQC method, it just spares time thanks to the limitation of the number of computation frequencies. The ratio of computational times is thus given by

$$
\frac{t_{N E W}}{t_{C Q C}} \simeq \frac{(M+1) \frac{M(M-1)}{2}}{n_{f} \frac{M(M-1)}{2}}=\frac{1}{40} \frac{M+1}{M}
$$

which makes the new method much faster than the strict application of the random vibration theory. 


\section{Example}

The influence of modal correlations is illustrated and the new relation is validated with a finite element analysis of the famous Viaduct of Millau (Barre 1999, Virlogeux 2003). With its pylons dominating the Tarn valley about $350 \mathrm{~m}$ above the ground, this exceptional 7-span cable-stayed bridge (approx. $2.5 \mathrm{~km}$ long) is the highest bridge ever built. The famousness of this bridge is not only dedicated to its final shape and design but also to the erection technique. Indeed in order to limit the risks undertaken during its construction, it was decided to opt for a deck launching on temporary piers.

The bridge is modelled with a finite element software for structural applications (FineLg 2003). The structure is represented with 2439 3-D beam finite elements (de Ville de Goyet 1995) (1425 nodes with 6 degrees of freedom each, leading thus to $N=8550$ degrees of freedom). The particular design, with seven spans of equal lengths, generates many close natural frequencies. And, because of the flexibility of the bridge, this eigen mode demultiplication leads to 40 modes between $0.17 \mathrm{~Hz}$ and $1 \mathrm{~Hz}$ ! Table 1 gives a brief description of the major modes.

The proximity of natural frequencies and the similarity of mode shapes (see Fig. 2) for modes 17 and 19 let guess a susceptibility to high modal correlation, which is discussed in the following.

The statistical characteristics of the wind model are listed in Table 2. They have been identified from on-site measurements. According to codes ruling the design of bridges subjected to wind

Table 1 Natural frequencies and brief description of major modes

\begin{tabular}{ccl}
\hline \hline Mode & $f_{m}=\frac{\omega_{m}}{2 \pi}[\mathrm{Hz}]$ & \\
\hline 1 & 0.174 & Out of plane bending of piles (and pylons) 2 and 3 \\
2 & 0.199 & In-plane bending of the deck and of the piles \\
3 & 0.221 & Out of plane bending of piles (and pylons) 2,3 and 4 \\
4 & 0.250 & In-plane bending of the deck \\
17 & 0.531 & In-plane bending of pile 3 \\
19 & 0.534 & In-plane bending of pile 3 and of the deck \\
\hline
\end{tabular}
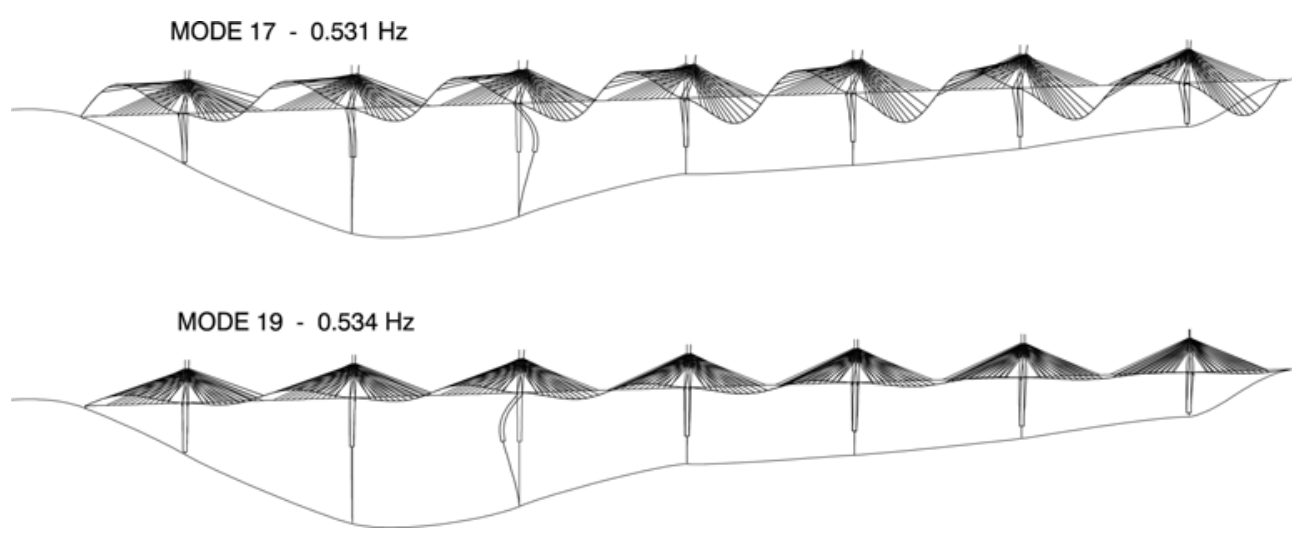

Fig. 2 Modes 17 and 19 have close natural frequencies and mode shapes. The correlation between these modes is studied in the example 
Table 2 Statistical characteristics of the wind loading

\begin{tabular}{|c|c|c|c|}
\hline & Zone A & Zone B & Zone $\mathrm{C}$ \\
\hline Reference Mean wind velocity $[\mathrm{m} / \mathrm{s}]$ & 38 & 34 & 36 \\
\hline Std of turbulence components $[\mathrm{m} / \mathrm{s}]$ & $6.5 / 6.5 / 4.5$ & $5.5 / 5.5 / 4.0$ & $5.5 / 5.5 / 4.0$ \\
\hline \multicolumn{4}{|l|}{ \# Longitudinal turbulence } \\
\hline Length Scales (Lx, Ly, Lz) [m] & $250 / 90 / 90$ & $250 / 90 / 90$ & $250 / 90 / 90$ \\
\hline Coherence Coefficients $(\mathrm{Cy}, \mathrm{Cz})$ & $12 / 12$ & $12 / 12$ & $9 / 9$ \\
\hline \multicolumn{4}{|l|}{ \# Vertical turbulence } \\
\hline Length Scale (Lx, Ly, Lz) [m] & $70 / 100 / 100$ & $70 / 100 / 100$ & $70 / 100 / 100$ \\
\hline Coherence Coefficients $(\mathrm{Cy}, \mathrm{Cz})$ & $12 / 12$ & $12 / 12$ & $9 / 9$ \\
\hline \multicolumn{4}{|l|}{ \# Transverse turbulence } \\
\hline Length Scale (Lx, Ly, Lz) [m] & $30 / 40 / 40$ & $30 / 40 / 40$ & $30 / 40 / 40$ \\
\hline Coherence Coefficients $(\mathrm{Cy}, \mathrm{Cz})$ & $12 / 12$ & $12 / 12$ & $9 / 9$ \\
\hline Wind profile & & Measured on site & \\
\hline Power Spectral density & & Measured on site & \\
\hline
\end{tabular}

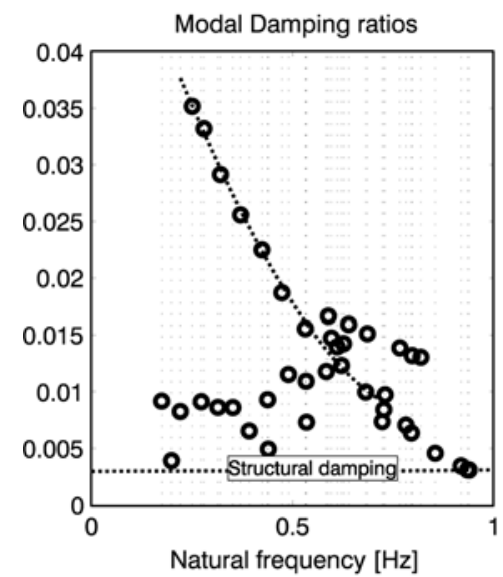

(a)

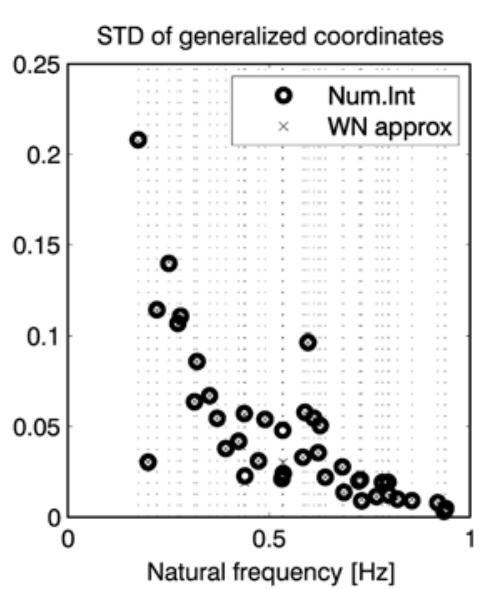

(b)

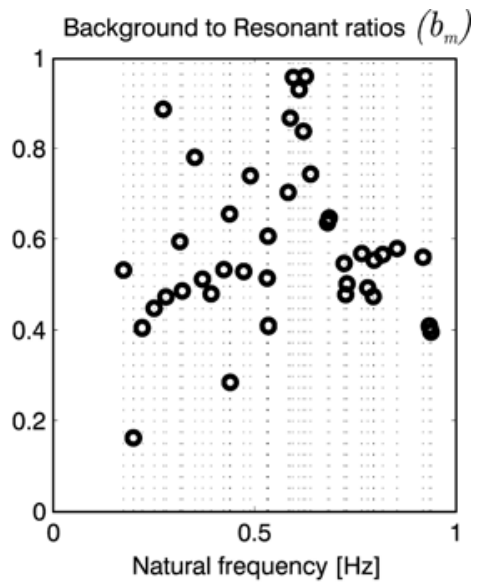

(c)

Fig. 3 Modal characteristics of the structure

forces (e.g., Eurocode 1), these characteristics are defined for a central zone (B) and two backside zones (A and $\mathrm{C}$ ). Based on these data and on wind tunnel measured aerodynamic coefficients, the PSDM of the wind forces are established (Cremona 2002). Together with the structural mode shapes resulting from the structural analysis, these are the necessary data to strictly apply the random vibration theory (see Section 1). A reference result (CQC) is obtained by following the steps presented in Section 1 and integrating numerically the PSDM of modal responses. The SRSS and proposed methods are both compared to this reference result.

Fig. 3(a) represents the modal damping ratios. They are the sum of:

- a structural damping common to each mode $(\xi=0.3 \%)$,

- an aerodynamic damping obtained from the mean wind velocity and aerodynamic coefficients (quasi-steady wind model). This damping is evaluated in a modal space and depends thus of the mode shapes. For example, it can be seen that in-plane bending modes are aerodynamically 
more damped than the other ones (upper dashed line).

Fig. 3(b) represents the standard deviations of the modal responses in the first 40 modes. Two results are presented. The rigorous approach provides numerically integrated results (circles) used for the CQC method. Because of the important sharpness of PSDs, a non uniform distribution of the integration points is used to reduce the number of computation frequencies (Denoël 2005). Values represented by crosses result from the decomposition into background and resonant contributions (Eq. (15)). They are used for the SRSS and the proposed methods. The good matching between both results is due to the low damping and the low frequency content of the loading. The error is lower than $1 \%$ except for modes 17 to 19 (3\% to 5\%). This plot indicates also that modal convergence can be considered to be reached with 40 modes.

The new method is based on the background-to-resonant ratio to interpolate between two limit correlations. They are represented in Fig. 3(c). These ratios $\left(b_{n}\right)$ are in good accordance with the order of magnitude reported by Gu et al. (2002).

Fig. 4 shows both limit correlations:

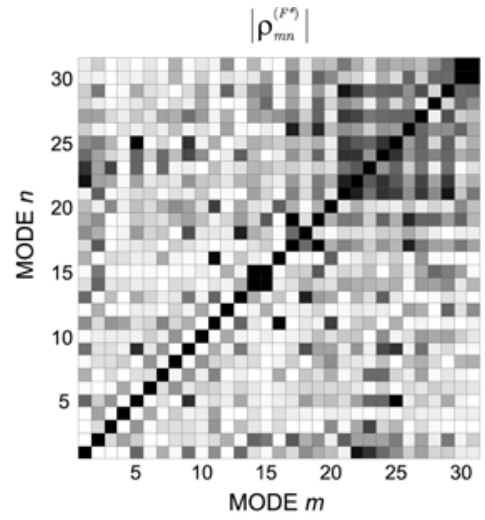

(a)

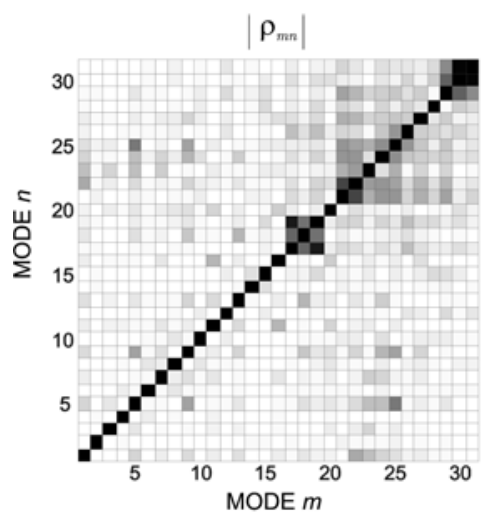

(c)

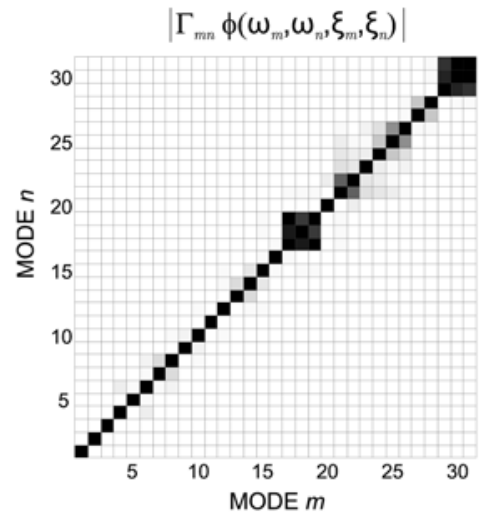

(b)

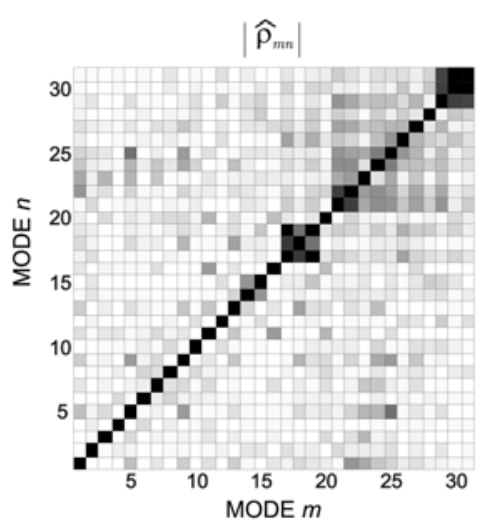

(d)

Fig. 4 (a) Correlation coefficients of the generalized forces, (b) Dynamic correlation coefficients, (c) Correlation coefficients of modal responses obtained by numerical integration of the corresponding PSDM (CQC approach), (d) Correlation coefficients of modal responses obtained with the simplified proposed relation 
- (a) the correlation coefficients of the generalized forces. They are equal to zero (in practical application, very small) when the mode shapes correspond to vibrations in orthogonal planes; and they are larger when the mode shapes are similar (e.g., 17, 19). If the structure is very stiff or highly damped, the correlation coefficient of the generalized coordinates is equal to this value.

- (b) the dynamic coefficients defined by Eq. (34). They are related to the relative (influenced by damping ratios) proximity of the natural frequencies $(\phi)$ and to the coherence of generalized forces at natural frequencies $\left(\overline{\Gamma_{m n}}\right)$. Both aspects are important. For example, the dynamic correlation coefficient (b) is larger for modes 17 and 19 than for modes 17 and 18. This could not be explained by considering the proximity of natural frequencies only; the reason is a higher coherence in the generalized forces for modes 17 and 19 than for modes 17 and 18. If the structure was very flexible (and slightly damped) the response would be mainly dynamic and the modal correlation coefficients would be equal to these quantities.

Real structures are not perfectly stiff or perfectly flexible. The actual modal correlation coefficients lie somewhere between or below these two extremes, since $\gamma_{B}+\gamma_{R}$ is slightly smaller than 1. Fig. 4(d) represents the correlation coefficient obtained with the proposed relation. They have to be compared to those of Fig. 4(c), resulting from the strict application of the random vibration theory. The good matching indicates that the simplified procedure gives a very good representation of the exact correlation coefficients, with significantly lighter computational efforts. The highest discrepancy concerns modes 17 to 19, for which even the decomposition of the variance into background and resonant contributions is the less accurate. Fig. 5 represents the absolute value of the difference between these results. The shaded representation (left) indicates that the difference remains very small. The correlation between modes 17 and 19 is the worst represented couple. The histogram of the difference of correlation coefficients (right) shows that the deviation from the exact result is usually smaller than 0.05 . The mean deviation is

$$
\frac{1}{M^{2}} \sum_{m=1}^{M} \sum_{n=1}^{M}\left|\rho_{m n}-\hat{\rho}_{m n}\right|=0.0148
$$

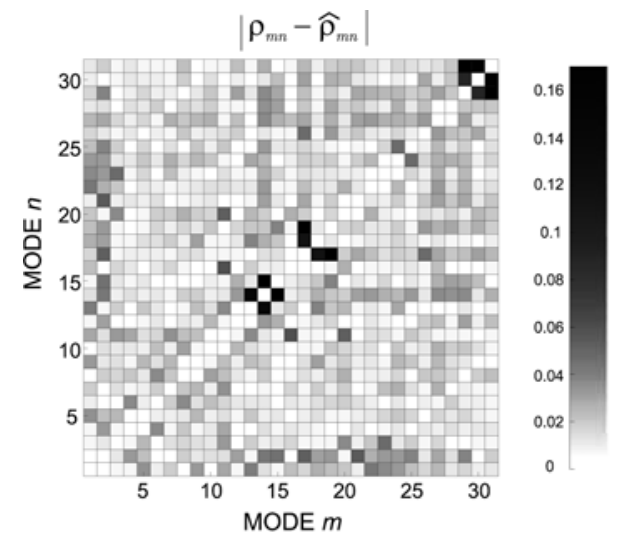

(a)

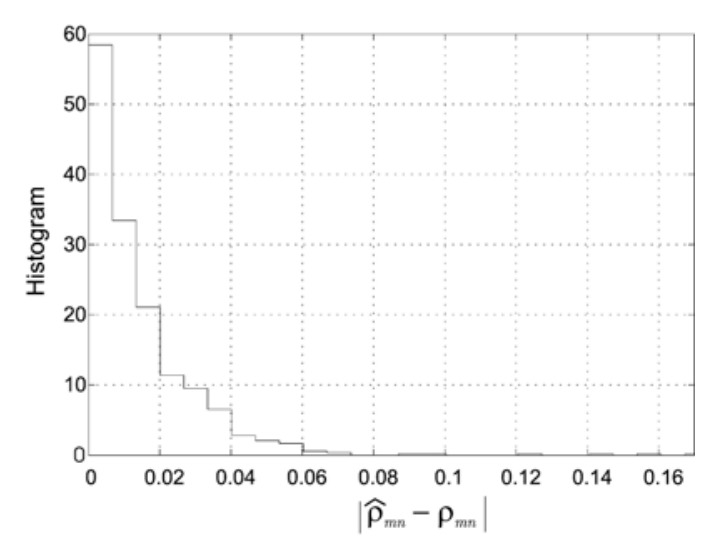

(b)

Fig. 5 Diffierence between the correlation coefficients obtained with the CQC approach and the proposed method - Shaded representation of all values (left) and histogram obtained for the first 40 modes (right) 

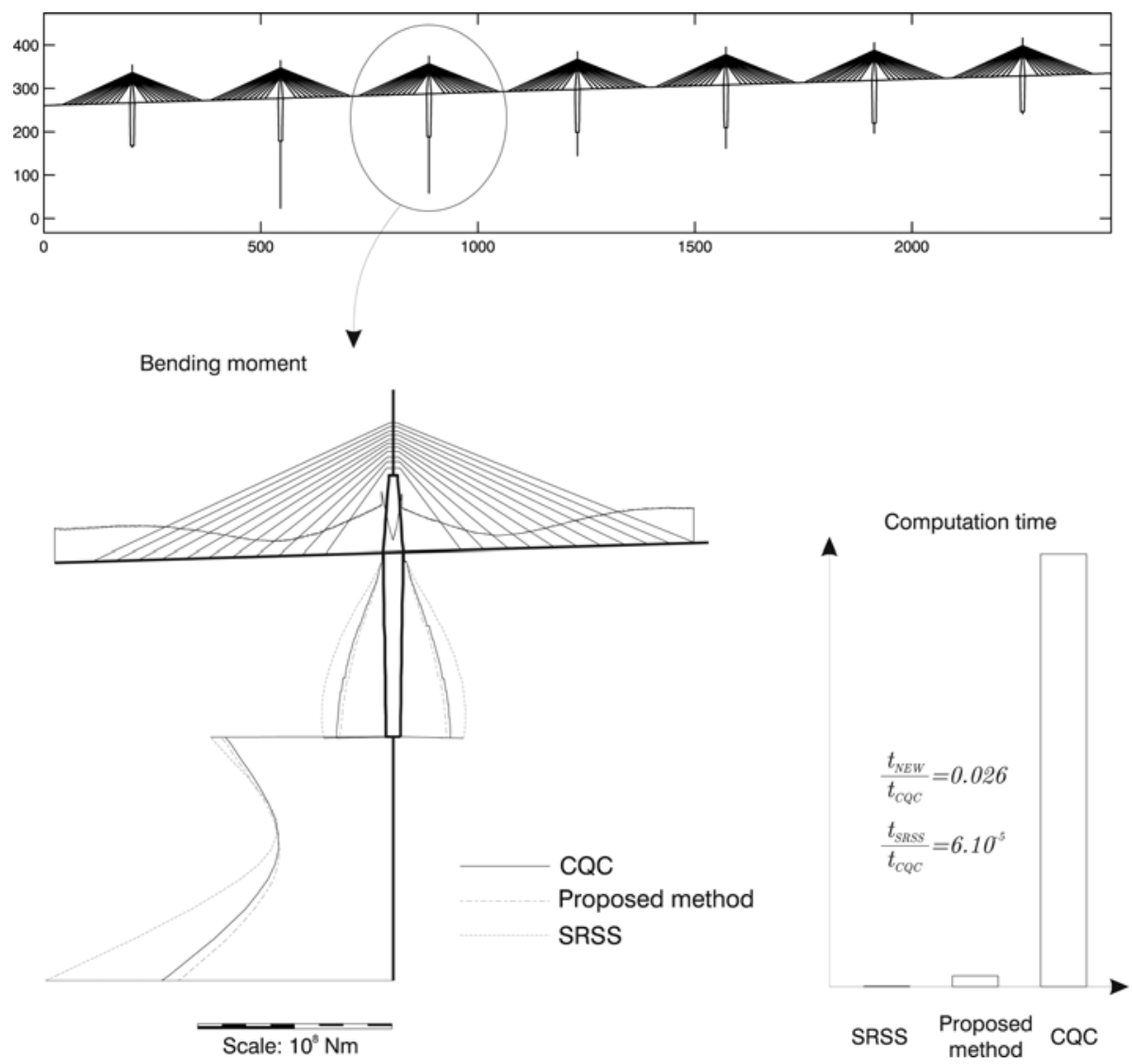

Fig. 6 In-plane bending moment diagram (standard deviations) obtained with the CQC, SRSS and the proposed method

Even if it does not give an accurate value of the correlation coefficient in modes 17 and 19, the simplified method predicts a high correlation between them. Since these modes involve the bending of the third pile, some differences can be expected in this part of the structure when the correlations between modal responses are considered or not. This is illustrated in Fig. 5 which represents the standard deviations of the in-plane bending, with and without modal cross-correlations (CQC and SRSS) and with the proposed method. The computational efforts are also schematically represented. In the bridge deck, all three methods give approximately the same result. At the foot of the pile, the bending moments obtained with each method are

$$
\begin{gathered}
M_{C Q C}=1.2010^{8} \mathrm{Nm} \\
M_{N E W}=1.1110^{8} \mathrm{Nm}(-7.5 \%) \\
M_{S R S S}=1.7810^{8} \mathrm{Nm}(+48 \%)
\end{gathered}
$$

which quantifies rigorously the difference between the different methods. The result provided by the proposed method is a worst case scenario, since the modal correlation between modes 17 and 19 is the less accurate. This example demonstrates that the proposed method is a good compromise between accuracy and computation time. 


\section{Conclusions}

The classical decomposition of the variances of modal responses into background and resonant contributions is used to give a convenient estimation of modal correlations. They are represented by a simple mathematical relation (Eq. (34)) and give therefore an interesting physical explanation to the origin of the modal correlation. The modal correlations can come from the background or resonant component, considered as extreme cases. In the first case, the modal correlation coefficients are equal to those of the generalized forces, whereas they are equal to a defined dynamic correlation coefficient in the second case (Eq. (34)).

The proposed relation provides an affordable access to a combination technique including correlation. It is however much more computationally efficient than the CQC because it avoids the discretization of the frequency space with a large number of frequencies. The proposed relation has to be seen as an intermediate solution between the CQC and SRSS techniques, as a compromise between accuracy and flexibility.

\section{Acknowledgements}

The work described in this paper was financially supported by the Belgian National Fund for Scientific Research. Sincere thanks are going the Prof. V. de Ville de Goyet (Greisch Ingénierie). Being involved in the design of the Millau viaduct, and consequently crackbrained by hours, days and weeks of buffeting analyses computations, he fed my motivation to establish this simplified procedure. The valuable exchange with Dr. H. Degée has to be underlined too. Many thanks finally go to Ing. C. Braham and Dr. T. Richard for the final touches to the organisation of this document.

\section{References}

Barre, C., Flamand, O. and Grillaud, G. (1999), “The Millau viaduct - Special wind studies for an exceptional structure", Wind Engineering Into the 21st Century, 1-3, 833-836.

Carassale, L. and Solari, G. (2006), "Monte Carlo simulation of wind velocity fields on complex structures", $J$. Wind Eng. Ind. Aerod., 94(5), 323-339.

Chen, X.H., Matsumoto, M. and Kareem, A. (2000), "Aerodynamic coupling effects on flutter and buffeting of bridges", J. Eng. Mech., ASCE, 126(1), 17-26.

Chen, X.Z. and Kareem, A. (2002), "Advances in modeling of aerodynamic forces on bridge decks", J. Eng. Mech., ASCE, 128(11), 1193-1205.

Chen, X.Z. and Kareem, A. (2004), "Equivalent static wind loads on buildings: New model", J. Struct. Eng., ASCE, 130(10), 1425-1435.

Chen, X.Z. and Kareem, A. (2006), "Revisiting multimode coupled bridge flutter: Some new insights", J. Eng. Mech., ASCE, 132(10), 1115-1123.

Chen, X.Z., Kareem, A. and Matsumoto, M. (2001), "Multimode coupled flutter and buffeting analysis of long span bridges", J. Wind Eng. Ind. Aerod., 89(7-8), 649-664.

Clough, R.W. and Penzien, J. (1993), Dynamics of Structures, McGraw-Hill, New-York.

Costa, C., Borri, C., Flamand, O. and Grillaud, G. (2007), "Time-domain buffeting simulations for wind-bridge interaction", J. Wind Eng. Ind. Aerod., 95, 991-1006.

Davenport, A.G. (1961), "A statistical approach to the treatment of wind loading on tall masts and suspension bridges", University of Bristol, Bristol.

Davenport, A.G. (1962), "The response of slender, line-like structures to a gusty wind", Proc. Inst. Civil Eng., 
23, 389-408.

de Ville de Goyet, V. (1995), "An advanced non-linear spatial beam finite element", Proceeding of the International Conference on Structural Stability and Design, Sidney.

Denoël, V. (2005), "Application of stochastic analysis methods to the study of the effects of wind on civil engineering structures (in french)", University of Liège, Liège.

Denoël, V. and Degee, H. (2006), "Influence of the non-linearity of the aerodynamic coefficients on the skewness of the buffeting drag force", Wind Struct., 9(6), 457-471.

Ding, Q.S., Chen, A.R. and Xiang, H.F. (2002), "Coupled buffeting response analysis of long-span bridges by the CQC approach", Struct. Eng. Mech., 14(5), 505-520.

FineLg. (2003), Non linear finite element analysis program: User's Manual, Unveristy of Liège, ArGEnCo and Greisch Ingènierie.

Gu, M., Chen, S.R. and Chang, C.C. (2002), "Background component of buffeting response of cable-stayed bridges", J. Wind Eng. Ind. Aerod., 90(12-15), 2045-2055.

Gurley, K.R., Tognarelli, M.A. and Kareem, A. (1997), "Analysis and simulation tools for wind engineering”, Prob. Eng. Mech., 12(1), 9-31.

Holmes, J.D. (2007), Wind Loading on Structures, SponPress, London.

Jain, A., Jones, N.P. and Scanlan, R.H. (1996a), "Coupled aeroelastic and aerodynamic response analysis of long-span bridges", J. Wind Eng. Ind. Aerod., 60(1-3), 69-80.

Jain, A., Jones, N.P. and Scanlan, R.H. (1996b), "Coupled flutter and buffeting analysis of long-span bridges", $J$. Struct. Eng., ASCE, 122(7), 716-725.

Katsuchi, H., Jones, N.P., Scanlan, R.H. and Akiyama, H. (1998), "Multi-mode flutter and buffeting analysis of the Akashi-Kaikyo bridge", J. Wind Eng. Ind. Aerod., 77-8, 431-441.

Preumont, A. (1994), Random Vibration and Spectral Analysis, Kluwer Academic Publishers.

Rossi, R., Lazzari, M. and Vitaliani, R. (2004), "Wind field simulation for structural engineering purposes", Int. J. Numer. Meth. Eng., 61(5), 738-763.

SAP2000. (2007), Integrated Software for Structural Analysis and Design: User's Manual (V. 11.0), CSI, Computers and Structures, Inc.

Scanlan, R.H. (1978), "Action of flexible bridges under wind. 2. Buffeting theory", J. Sound Vib., 60(2), 201211.

Scanlan, R.H. and Jones, N.P. (1990), “Aeroelastic analysis of cable-stayed bridges”, J. Struct. Eng., ASCE, 116(2), 279-297.

Simiu, E. and Scanlan, R. (1996), Wind Effects on Structures, John Wiley and Sons.

Virlogeux, M. (2003), "The Millau cable-stayed bridge", Recent Developments in Bridge Engineering, 3-18.

Zienkiewicz, O.C. and Taylor, R.L. (1991), The Finite Element Method, McGraw-Hill, U.K. 